\title{
LOS JUEGOS Y SU ROL EN EL APRENDIZAJE DE UNA LENGUA
}

\author{
Lía Trinidad Castrillón Díaz
}




\section{GAMES AND THEIR ROLE IN LANGUAGE LEARNING}

\section{ABSTRACT}

This article discusses how games can have a positive influence on the acquisition of a second language based on theorists who have studied its importance in both the teaching and learning of a new language. Learning a second language is vital in our society and thanks to the implementation of the games in the classroom, teachers have a motivational tool which allows their students to express their feelings in a playful way while creating a better environment in the classroom. In the light of this, undergraduate students at Universidad Autónoma de Bucaramanga- UNAB- can enhance learning the second language with the use of games not only in the classroom but also outside it.

Key words: Second language, game, playful.

\section{RESUMEN}

Este artículo plantea cómo los juegos pueden tener una influencia positiva en la adquisición de un segundo idioma con base en los teóricos que han estudiado su importancia tanto en la enseñanza y el aprendizaje de un nuevo idioma. El aprendizaje de una segunda lengua es de vital importancia en nuestra sociedad y gracias a la implementación de los juegos en el aula, los profesores tienen una herramienta de motivación que permite a sus estudiantes expresar sus sentimientos de una manera lúdica, mientras que se crea un mejor ambiente en el salón de clase. A la luz de esto, los estudiantes de pregrado de la Universidad Autónoma de Bucaramanga - UNAB pueden mejorar el aprendizaje de la segunda lengua con el uso de juegos no sólo en el aula sino también fuera de ella.

Palabras clave: Segunda lengua, juego, lúdica.

\section{AUTOR}

Lía Trinidad Castrillón Díaz

Master in English Language teaching for Self-directed Learning. Universidad de la Sabana

Docente Universidad Autónoma de Bucaramanga

Correo electrónico: Icastrillon@unab.edu.co

Recibido: 15/05/2017

Aprobado: 01/06/2017 
"El juego es una experiencia creadora. $Y$ es una experiencia en el continuo espacio-tiempo. Una forma de vida"

Lev S. Vigotsky

A través de la historia los hombres han encontrado en el juego una manera de cambiar de actitud y de superar muchas dificultades que se les presentan diariamente. Desde el bebé que es inducido al juego por sus padres con el uso del sonajero $u$ otros elementos infantiles hasta el adulto que sin darse cuenta juega con el lapicero, se aprecia como éste hace parte del ser humano en sus diferentes estadios de vida, ya sea de manera consciente o inconsciente. En tal sentido, quién no recuerda sus juegos infantiles en la casa, tales como: el domino, el parques, las damas, el twister o hágase rico, en los cuales se necesitaba tener paciencia, tolerancia, conocer y aprender cómo se juega. Quién no recuerda en el colegio o en el parque el estar jugando baloncesto, voleibol, futbol o maras y tener que manejar los sentimientos cuando era empujado o lastimado por un compañero del equipo contrario. Quién no recuerda como estos juegos le ayudaron a manejar los sentimientos al perder o quedar empatado ante su rival y como le enseñaron a seguir normas y reglas preestablecidas. Crespillo (2010) afirma que el juego ayuda al ser humano a controlar el cuerpo, los sentimientos y resolver problemas emocionales para que pueda ser un ser social y aprender a ocupar un lugar dentro de la comunidad.

Algunos juegos han pasado de generación en generación y por ello se han vuelto populares. Según la Real Academia de la Lengua, el juego se define como "el ejercicio recreativo o de competición sometido a reglas, y en el cual se gana o se pierde". De acuerdo con la declaración de los derechos del niño hecha por Naciones Unidas en 1959 el juego es un derecho fundamental del niño y por otra parte en la Constitución Política de Colombia, en el artículo 52, declara el derecho de todas las personas a la recreación, a la práctica del deporte y al aprovechamiento del tiempo libre.

Teniendo en cuenta lo anterior, la implementación de los juegos en la educación hace del aula un lugar lúdico de aprendizaje y de motivación constante para muchos estudiantes. De ahí que la utilización de estos en el salón de clase ha influido de manera positiva en el aprendizaje, aunque esta aplicación sea muy nueva en el ámbito educativo. Juan Moreno Murcia citado en García (2007a) habla del estudio del juego teniendo en cuenta su origen, evolución, aportes sociales, aplicaciones y orientaciones en la educación infantil y concretamente en el diseño curricular de primaria para facilitar su aplicación en la enseñanza. Por otra parte, Rheta (1980) manifiesta la importancia de un tipo de juegos, los colectivos, en los cuales los niños pueden aprender y el maestro puede alcanzar lo máximo del aprendizaje con su implementación teniendo en cuenta

la teoría de Piaget y la pedagogía constructivista. Para ello, hay que tener en cuenta que Piaget (como se citó en Banda, 2008) habla de tres estructuras básicas del juego con las fases evolutivas del pensamiento humano: el juego como simple ejercicio (parecido al anima); el juego simbólico (abstracto, ficticio); y el juego reglado (colectivo, resultado de un acuerdo de grupo). Los juegos de reglas permiten que los jugadores tengan exigencias para alcanzar la victoria o la derrota y además manejar la competitividad. De esta manera el juego ayuda al niño y al joven a adecuarse a la sociedad de una manera lúdica.

Algunos teóricos han mencionado la importancia del juego no sólo en la parte educativa sino formativa 
del ser. Groos (2012a) fue el primero en relacionar el juego con el desarrollo del pensamiento y la actividad; permitiendo que el adulto esté preparado para su vida. El filósofo y psicólogo Groos (2012b) habla de una teoría de la función simbólica, es decir que de un juego previo saldrá un comportamiento posterior. Lo anterior basado en estudios de Darwin en donde se indica que las especies que sobreviven son las mejor adaptadas a las condiciones cambiantes del medio. De esta manera el juego es una preparación para la vida adulta y la supervivencia. Es por ello, que los docentes que implementen juegos dentro de su clase a nivel escolar y universitario ayudarán a sus estudiantes a adaptarse mejor a su medio y les permitirán tener una mejor vida profesional, gracias a todo lo que un juego involucra.

Por otra parte, Piaget (2012) menciona que el juego hace parte de la inteligencia de los niños y forma parte de la etapa evolutiva del individuo. Es decir, tiene relación con la madurez física y la experiencia de cada ser porque a través de ella es como se adquiere y se comprende el conocimiento.

Para Vigotsky (2011), el juego nace como la necesitad de interactuar con el otro. Por eso el juego es considerado dentro de su teoría como una actividad social, que permite establecer roles que son complementados con los propios. Vigotsky destaca dos fases evolutivas significativas sobre el juego en las fases evolutivas infantiles:

- De 2 a 3 años en la que los niños entienden la función real simbólica de los objetos.

- De 3 a 6 años en la que los niños hacen una representación imitativa.

Después de que el niño crece el juego dramático, es decir, la representación teatral y musical será un excelente recurso psicopedagógico que puede ayudar en el desarrollo de las habilidades afectivas y comunicativas. De ahí que el incluir en las clases de inglés las dramatizaciones, realizaciones de videos, concurso de canto, etc. Ayudará a los estudiantes a construir su aprendizaje y su propia realidad social y cultural.

Robert(2011) afirma que hay una relación entre el tipo de valores inculcados por una cultura y la clase de juegos que son promovidos por cada cultura. De ahí que se hable de una teoría de la enculturación que identifica los juegos de una cultura como medio para asegurar la transmisión de sus valores e ideologías propias.

Si se quiere lograr que el juego trascienda dentro de una cultura es necesario tener en cuenta las características que Pastor (2004) menciona para que el juego sea efectivo. Por ello, a la hora de organizar juegos en el salón de clase se debe cumplir con lo siguiente:

- Tener normas para que cumpla su objetivo.

- Tener objetivos claros y concretos.

- Tener una actividad que permita definir hasta qué punto va el juego.

- El profesor debe permitir la libertad en el desempeño y cambiar su rol de supervisión.

- El profesor debe hacer que sea divertido y atractivo para los estudiantes.

El aprendizaje del inglés en este mundo globalizado es de vital importancia debido a que contribuye a propiciar en los estudiantes oportunidades de integración y reconocimiento de nuevas culturas, junto con la motivación por conocer más el mundo y crear vínculos de amistad o laborales entre otros; la implementación de estrategias para el aprendizaje del mismo en el ámbito educativo se hace indispensable. 
Es de conocimiento popular que el aprendizaje del inglés tiene un impacto no sólo a nivel educativo sino comercial y social de ahí, la necesidad de buscar nuevas estrategias que aumenten la motivación para su aprendizaje, el cual es considerado difícil y una meta muy alta de alcanzar en algunos sectores vulnerables de la sociedad.

Es por ello que la implementación del juego dentro del aula de clase y en el aprendizaje de una lengua logra producir una mayor motivación extrínseca e intrínseca y puede llegar a ser una herramienta excelente para practicar estructuras gramaticales, lingüísticas y adquirir conocimientos socioculturales de la lengua que se esté aprendiendo. Malgren (2011) define el juego como un cambio a la enseñanza tradicional donde la creatividad y la imaginación de los estudiantes, es focalizada en situaciones de aprendizaje facilitando con ello la adquisición de una lengua. A través del juego los estudiantes aprenden a escuchar, expresar opiniones y tomar decisiones propias.

La implementación de la actividad lúdica en el salón de clase se concibe como aquella que tiene en cuenta el juego, visto como una herramienta de aprendizaje, el cual tiene reglas, su propia dinámica, e involucra a los participantes quienes deben alcanzar un fin. Por ello, el juego pretende ayudar a muchos estudiantes y maestros a potenciar el aprendizaje de la segunda lengua, de tal manera que unos y otros interactúen no sólo a través de la lengua, sino de la cultura que ella misma involucra.

Cuando se usa un juego en la clase, el estudiante se transforma y cambia su conducta de aprendizaje, mostrándose más relajado, confiado, desinhibido y con grandes deseos de aprender para ganar. Castañeda (2010) menciona que el juego puede ser utilizado por el maestro dentro del ambiente escolar, como una herramienta o estrategia de enseñanza-aprendizaje, para cualquier saber específico (disciplina) facilitando de una u otra el quehacer del maestro, pues el juego no es una actividad que requiera muchos elementos, tan solo una gran creatividad e imaginación por el ejecutor, en este caso el maestro, convirtiéndose en el más efectivo y practico medio de motivación hacia el aprendizaje de los niños y jóvenes.

Malgren (2011) afirma que una de las maneras que tienen los profesores para ayudar a sus estudiantes en adquirir una lengua nueva es el juego como método de aprendizaje y esto puede consistir en actuar, jugar y cantar. De ahí que el juego facilite el aprendizaje. Los estudiantes adquieren el conocimiento de la segunda lengua fijando su conocimiento en la mente muchas veces sin describir la estructura, ni la gramática. Este es un método que ayuda a todos los estudiantes porque es divertido y menos formal. Es trascendental que los docentes de inglés incluyan en sus clases desde preescolar hasta la universidad diferentes juegos que de acuerdo con la edad de sus estudiantes y los tópicos estudiados en clase, les faciliten el aprendizaje de la misma.

Lozano (2014) asevera que el implementar juegos en el aula de clase, es fundamental para el desarrollo y la educación de los alumnos en un idioma extranjero porque supone mayor motivación e interés; además permite la participación de los mismos de una manera desinhibida ayudándoles a una interiorización y asimilación de los conceptos de una manera más realista.

Otra ventaja es la mejoría del ambiente en la clase, el cual se torna relajado y divertido permitiendo el acercamiento del maestro a sus estudiantes y mejorando 
las relaciones sociales. De ahí que el filtro afectivo se reduce permitiendo que los estudiantes aprendan descubriendo que el error es parte del proceso de aprendizaje. Krashen (1982) quien es uno de los exponentes que ha tratado de los diversos aspectos que afectan el proceso de aprendizaje y fue quien propuso la hipótesis del filtro afectivo. Observó que el estado emocional de los alumnos y sus actitudes actúan como un filtro que les permite que entre la información necesaria para la comprensión, o bien pueda impedir o bloquear la información necesaria para el aprendizaje del idioma. Es decir, cuanto más alto sea el filtro afectivo-reflejado en un mayor nivel de ansiedad, baja autoestima y poca participación- más serán las posibilidades de que el estudiante fracase en el proceso de aprendizaje de una lengua. Pero, un filtro afectivo bajo le permitirá un mejor nivel de adquisición y aprendizaje de una segunda lengua. Krashen en su teoría de filtro afectivo abarca los aspectos de motivación, la actitud, la ansiedad y la autoconfianza para identificar si el proceso de adquisición de la lengua es positivo o negativo. Por ello es significativo que el docente de inglés incluya dentro de las clases diferentes juegos con situaciones comunicativas reales, permitiendo la participación e integración de los estudiantes con el fin de reducir el filtro afectivo. De esta manera los estudiantes de pregrado de la UNAB que llegan con nivel A1 o -A1 según el marco común europeo podrán mejorar su proceso de aprendizaje de la segunda lengua y superar sus dificultades. A su vez, los más aventajados podrán encontrar una manera lúdica de afianzar sus conocimientos y ponerse retos en su aprendizaje.

Por otra parte, Zapata (1997) reconoce la importancia de involucrar el juego en los sistemas educativos, específicamente en América Latina, por los aportes significativos que ella considera, las actividades lúdicas son un instrumento de conocimiento, un factor de socialización, regulador y compensador de la efectividad, instrumento del desarrollo de las estructuras del pensamiento en el niño y medio esencial de organización, desarrollo y afirmación de la persona infantil.

García (2007b) manifiesta que el aprender el inglés en un espacio lúdico-didáctico además de fortalecer, permite que los niños se habitúen a la segunda lengua, se identifiquen con ella y se sientan más seguros al demostrar sus habilidades, la valoren, disfruten y reconozcan las oportunidades que tendrán con relación a su desarrollo profesional, intercultural y calidad de vida.

Además, García (2007c) menciona que "el juego es una estrategia pedagógica múltiple y significativa, porque contribuye al aprendizaje de la nueva lengua y al desarrollo pluridimensional del individuo, favoreciendo los valores, el trabajo en equipo, fortaleciendo las emociones; incentivando la creatividad y los niveles de concentración." Otro aspecto positivo que menciona la autora es "el reconocimiento de los valores como la justicia y las consecuencias del incumplimiento a las normas, lo cual ayuda a los niños en su desarrollo social permitiéndoles una preparación futura para sus vidas." En conclusión, la implementación de juegos en la clase de inglés ayudará a chicos y a grandes a mejorar su proceso de enseñanza aprendizaje, permitiéndoles cambiar de actitud hacia la segunda lengua y redescubriendo sus potencialidades hacia la misma. Los estudiantes de la UNAB con esta estrategia encontrarán una manera lúdica de superar dificultades y de afianzar sus conocimientos logrando así un acercamiento mayor al idioma y reduciendo su filtro afectivo. De esta manera el juego es un medio para alcanzar objetivos claros dentro de la enseñan- 
za del inglés. Por ello, sería conveniente que todos los docentes dedicados a la enseñanza de una segunda lengua se dieran la oportunidad de utilizar esta estrategia como parte del proceso de enseñanza aprendizaje.

\section{REFERENCIAS}

Crespillo, E. A. (Agosto de 2010). El juego como actividad de enseñanza- aprendizaje. Recuperado el 4 de agosto de 2016, de GIBRALFARO ESTUDIOS PEDAGOFICOS: http://www.gibralfaro.uma.es/educacion/pag_1663.htm

Banda, E. M. (15 de Noviembre de 2008). Monografías. com. Recuperado el 4 de agosto de 2016, de http:// www.monografias.com/trabajos65/uso-juego-estrategia-educativa/uso-juego-estrategia-educativa2.shtml

Castañeda, C. C. (24 de Noviembre de 2010).

Recuperado el 5 de agosto de 2016, de Docplayer: http://docplayer.es/7052421-La-ludica-como-recurso-para-la-ensenanza-del-ingles-en-el-grado-primero-consuelo-castano-castaneda.html

Española, R. A. (6 de agosto de 2016). Real Academia Española. Obtenido de http://dle.rae.es/?id=MaS6XPk García, E. V. (2007). El juego como estrategia pedagógica para el aprendizaje del inglés como lengua extranjera en los niños de quinto grado curso "B", del CEDIT Jaime Pardo Leal. Bogotá.

Groos, K. (12 de Noviembre de 2012). Teorias del Juego. Recuperado el 4 de agosto de 2016, de https://actividadesludicas2012.wordpress.com/2012/11/12/teorias-de-los-juegos-piaget-vigotsky-kroos/

Krashen, S. (1982). Principles and Practice in Second
Language Acquisition (Oxford: Pergamon, 1982). En S. Krashen, Principles and Practice in Second Language Acquisition (págs. 30-32). Oxford: Pergamon.

Lozano, A. P. (2014). Universidad de Valladolid. Recuperado el 6 de agosto de 2016, de https://uvadoc. uva.es/bitstream/10324/7789/1/TFG-G\%20895.pdf

Malgren, C. L. (2011). Akademin för utbildning, kultur och. Recuperado el 5 de agosto de 2016, de http:// www.diva-portal.se/smash/get/diva2:558137/FULLTEXT01.pdf

Pastor, C. S. (2004). Razones y objetivos de la enseñanza de la gramática. Aprendizaje de segundas lenguas. Alicante: Servicio de publicaciones de la Universidad.

Piaget, J. (12 de Noviembre de 2012). Teorias del Juego. Recuperado el 4 de agosto de 2016, de https://actividadesludicas2012.wordpress.com/2012/11/12/teorias-de-los-juegos-piaget-vigotsky-kroos/

Rheta, K. C. (1980). Juegos Colectivos en la Primera Infancia Implicaciones de la Teoría de Piaget. Madrid: Antonio Machado .

Robert, S. S. (diciembre de 2011). El juego en Educación Infantil. Recuperado el 4 de agosto de 2016, de http:// www.csi-csif.es/andalucia/modules/mod_ense/revista/pdf/Numero_6/ESTHER_HERVAS_1.pdf

Vigotsky, L. S. (5 de Enero de 2011). Revista electrónica de Educación. Recuperado el 4 de agosto de 2016, de http://biblioteca.ucm.es/revcul/e-learning-innova/5/ art382.php\#.V6gUGTvhDDe

Zapata, O. (1997). Juego y Aprendizaje Escolar. Perspectiva Psicogenética. Mexico : Editorial Pax México. 
Citar este artículo como: Castrillón, L. (2017). "Los juegos y su rol en el aprendizaje de una lengua". En: Revista La Tercera Orilla (19). Bucaramanga: Universidad Autónoma de Bucaramanga. 